\title{
Early experience with in-vivo optical coherence tomography for differentiating lesions of the upper aerodigestive tract Christian Stephan Betz ${ }^{* 1}$, Veronika Volgger ${ }^{1}$, Herbert Stepp ${ }^{2}$, Waseem Jerjes ${ }^{3}$, Tahwinder Upile ${ }^{3}$, Andreas Leunig ${ }^{1}$ and Colin Hopper ${ }^{3}$
}

\author{
Address: ${ }^{1}$ Department of Otorhinolaryngology, Head \& Neck Surgery, Ludwig Maximilian University Munich, Marchioninistr, Munich, Germany, \\ ${ }^{2}$ Laser-Research Laboratory, LIFE Center, Ludwig Maximilian University, Marchioninistr, Munich, Germany and ${ }^{3}$ Department of Oral and \\ Maxillofacial Surgery, University College London Hospital, Mortimer Market, London WC1E 6AU, UK \\ * Corresponding author
}

from Ist Scientific Meeting of the Head and Neck Optical Diagnostics Society

London, UK. 14 March 2009

Published: 28 July 2009

Head \& Neck Oncology 2009, I(SuppI I):OI5 doi:I0.I I86/I758-3284-I-SI-OI5

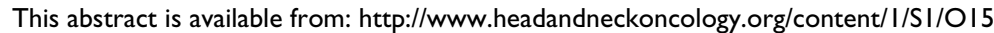

(c) 2009 Betz et al; licensee BioMed Central Ltd.

\section{Objective}

In vivo detection of dysplastic or early invasive mucosal changes is expected to greatly reduce both morbidity and mortality of oral and pharyngeal cancer. Optical Coherence Tomography (OCT) seems to be well suited for this application.

\section{Methods}

In this ongoing study, 28 patients with a total of 34 primary, flat mucosal lesions of the upper aerodigestive tract (OADT) were prospectively examined using a timedomain, in vivo OCT (Niris ${ }^{\circledR}$, Imalux Corporation, USA; lateral resolution $25 \mu \mathrm{m}$ /axial resolution $15 \mu \mathrm{m}$ ) and the results were compared to the histopathological reports on subsequent tissue biopsies from the same areas. Additionally, an intraoral screening was performed on 52 healthy volunteers.

\section{Results}

On the OCT images, surface structures such as the keratin and epithelial layer, the epidermal/dermal junction and areas of cellular crowding were clearly identifiable and showed a good correlation to the histopathological slides down to a depth of approximately $1.5 \mathrm{~mm}$. Of 34 lesions investigated so far, 2 out of 2 early malignant lesions as well as 29 out of 32 non-/premalignant lesions could be correctly differentiated using OCT. The screening resulted in a high degree of variability in the physiological thick- ness of intraoral epithelium (ø126 $\mu \mathrm{m}$ at the floor of mouth to $ø 487 \mu \mathrm{m}$ at the lateral border of tongue).

\section{Conclusion}

From these results, the method seems to hold great promise for early in vivo tumour diagnosis and depth measurement in early invasion. Further efforts are currently being undertaken for an enhancement of image quality and contrast. 